\title{
The Potential Targets of Chinese Medicines in the Treatment of Parkinson's Disease: Old Wine in New Bottles
}

\author{
Yongjin Zhang ${ }^{1}$, Jiaojiao $\mathrm{Li}^{1}, \mathrm{Na} \mathrm{Wang}^{1}$, Jingfeng Ming ${ }^{1}$, Zenglin Cai ${ }^{1}$ and Xiaomin $\mathrm{Li}^{2}$ \\ 1. Department of Neurology, Affiliated Lianyungang Hospital of Xuzhou Medical College, Lianyungang 222000, China \\ 2. Department of Emergency, Affiliated Lianyungang Hospital of Xuzhou Medical College, Lianyungang 222000, China
}

\begin{abstract}
This article systematically reviews the present research situation of the pathogenesis of PD (Parkinson's disease) and our recent research over the past decade, to discuss the potential targets of many TCM (traditional Chinese medicines) in prevention and treatment of $\mathrm{PD}$, such as against oxidative stress (Astragaloside, protocatechuic aldehyde, Liu-Wei-Di-Huang), anti-apoptosis (Geniposide, Zhichan powder), improve mitochondrial function (Paeoniflorin, Da-Bu-Yin-Wan), inhibition of protein aggregation (Panax ginseng, Irisflorentin), induce autophagy (piperine, Paeoniflorin), and many other mechanisms and multiple effectiveness (Saponins, Acanthopanax). With the research and development in PD pathogenesis and clarifying the targets of a variety of traditional Chinese medicine, especially its monomeric components, the development of traditional Chinese medicine in prevention and treatment of PD will have very broad application value and prospect.
\end{abstract}

Key words: Parkinson's disease, TCM (traditional Chinese medicines), oxidative stress, apoptosis, autophagy, protein aggregation.

\section{Introduction}

Initial characterization of the pathology in PD (Parkinson's disease) focused attention on the presence of the Lewy body in remaining dopaminergic neurons and the role played by melanin $[1,2]$. Current research indicates that multiple factors involved in the pathogenesis of $\mathrm{PD}$, including spontaneous process of cells, namely autophagy lysosome dysfunction [3], proteasome dysfunction, mitochondrial dysfunction [4]; non-spontaneous process of cells, such as neuroinflammatory reactions, apoptosis, oxidative stress and loss of nutritional support [5, 6]. There are many crossing signal system between the various molecular mechanisms [7, 8].

The bulk degradation of cytoplasmic proteins or organelles is largely mediated by macroautophagy, a process generally referred to as autophagy [6].

Corresponding author: Zenglin Cai, Dr., research fields: the role of autophagy, dynein in neurodegenerative diseases and clinical research of neurodegenerative diseases.
Autophagic vacuoles are formatted throughout the cytoplasm. The mature autophagy (autophagosome) retrograde transports to perinuclear regions where lysosomes are more abundant, then autophagosome fusion with lysosome to degrade aggregate-prone proteins [6].

Two thousand years ago, in the Chinese medical literature, Yellow Emperor had first described the symptoms of PD. TCM (traditional Chinese medicine) is a holistic system of medicine and has been used to treat many diseases for thousands of years in China. The earliest books on traditional Chinese medicine published about in the Han Dynasty, that is Shen Nong Ben Cao Jing [9].

With the deepening research of the pathological mechanism of PD, more and more Chinese and foreign scholars are concerned about a lot of Chinese medicine and its monomer composition in the mechanism of PD [10, 11], mainly involving against oxidative stress, improve mitochondrial function, improve nerve inflammation and immune response, reduce 
excitotoxic, anti-apoptosis, induce autophagy and inhibit the accumulation of abnormal proteins, etc. [12-14].

\section{Against Oxidative Stress}

Although PD etiology is not clear, and that is the result of multiple factors, oxidative stress and mitochondrial dysfunction play an important role on the occurrence and development of the disease. Free radicals and oxidative stress contribute to the cascade of events leading to dopamine cell degeneration in PD. Antioxidant therapy may represent an attractive strategy for treating or preventing the neurodegeneration $[15,16]$.

AS-IV (Astragaloside intravenous IV) can be extracted from the dried root of Astragalus membranaceus, a well-known Chinese medicine used for the treatment of neurodegenerative diseases, while Chan et al. [17] investigated its capacity to protect dopaminergic neurons in experimental PD. Their results showed that AS-IV can protect dopaminergic neurons against 6-OHDA-induced degeneration. Besides the neuroprotective effect, AS-IV alone promoted neurite outgrowth and increased $\mathrm{TH}$ (tyrosine hydroxylase) and NOS (nitric oxide synthase) immunoreactive of dopaminergic neurons. They concluded that the neuroprotective and neurosprouting effects of AS-IV are specific for dopaminergic neurons and it has therapeutic potential in the treatment of PD. Gao et al. [18] found that PAL (protocatechuic aldehyde), a traditional Chinese medicine compound, inhibited production of reactive oxygen species and the inhibition was abated in DJ-1-knockdown cells. PAL increased and decreased phosphorylation of AKT (serine/threonine kinase) and PTEN (phosphatase and tensin homolog deleted from chromosome 10), respectively, in SH-SY5Y cells. They demonstrated that PAL has potential neuroprotective effects through DJ-1. Results from Tseng et al. [19] revealed that LWDH (Liu-Wei-Di-Huang), a widely used TCM, possesses protection on dopaminergic neurons through enhancing antioxidant defense and decreasing apoptotic death, suggesting the potential benefits of LWDH-WE (Liu-Wei-Di-Huang-Wan) for PD treatment.

\section{Anti-apoptosis}

Apoptosis seems to play a key role in the progression of several neurologic disorders, like AD (Alzheimer's disease) and PD. Many dying neurons have been detected in brains of patients with neurodegenerative diseases, and these conditions are often associated with significant cell loss accompanied by typical morphological features of apoptosis, such as chromatin condensation, DNA (deoxyribonucleicacid) fragmentation, and activation of cysteine-proteases and caspases. Neurodegenerative diseases have been associated with reactive oxygen species and nitric oxide. Neuronal apoptosis and oxidative stress involve in the neurodegenerative diseases [20].

Geniposide, an active component of Gardenia jasminoides Ellis which is used in TCM, has shown neuroprotective and growth-factor, like effects in several in-vivo and in-vitro studies [21,22]. Chen et al. [21] concluded that Geniposide exerted its neuroprotective effect by enhancing growth factor signaling and the reduction of apoptosis in an acute PD mouse model induced by MPTP (1-methyl-4-phenyl-1,2,3,6-tetrahydropyridine) intraperitoneal injections, with few known side effects and shows potential as a drug treatment for PD. Zhichan powder diminished mRNA (messenger ribonucleic acid) and protein expression of tumor necrosis factor Receptor 1, Fas, Caspase-8, Cytochrome C, Bax, Caspase-3, and P53, but increased $\mathrm{Bcl}-2$ expression in the substantia nigra of rats with $\mathrm{PD}$, and exerts therapeutic effects on PD [23].

\section{Improve Mitochondrial Function}

Mitochondrial dysfunction exists in most neurodegenerative diseases. These disorders include bioenergetic defects, respiratory chain induced 
oxidative stress, mitochondrial dynamics defects, increased sensitivity to apoptosis and accumulation of damaged mitochondria. Despite significant progresses in the pathophysiology of mitochondrial disease have been achieved, there is still no effective treatment. The development of new metabolic treatments will be useful not only for rare mitochondrial disorders but also for the wide spectrum of common age-related neurodegenerative diseases shown to be associated with mitochondrial dysfunction [24].

PF (Paeoniflorin), a monoterpene glycoside isolated from the aqueous extract of Radix Paeoniae Alba, is widely used in TCM for treatment of neurodegenerative disorders, such as $\mathrm{AD}$ and PD. PF could protect $\mathrm{PC} 12$ cells against glutamate-induced injury in a concentration-dependent manner; It demonstrated that PF has neuroprotective effect on glutamate-induced apoptosis in PC12 cells via regulating mitochondrial membrane potential and Bcl-2/Bax signal pathway [25]. DBYW (Da-Bu-Yin-Wan), a historically traditional Chinese medicine formula, raised the mitochondrial mass, improved mitochondrial complex I activity and increased cellular ATP content, moreover, DBYW enhances the protective effect of DJ-1 medicated Akt phosphorylation on mitochondrial function [26].

\section{Inhibition of Protein Aggregation}

The aggregation of $\alpha \operatorname{Syn}(\alpha$-synuclein) leading to the formation of Lewy bodies is the defining pathological hallmark of PD [27]. $\alpha$-synuclein may contribute to PD pathogenesis in a number of ways, but it is generally thought that its aberrant soluble oligomeric conformations and termed protofibrils are the toxic species that mediate disruption of cellular homeostasis and neuronal death, through effects on various intracellular targets, including synaptic function. It is clear that $\alpha$-synuclein represents a valid therapeutic target in PD and possibly in related synucleinpathies [28].

Panax ginseng has been used in TCM for centuries.
Among its various benefits, a pluripotent targeting of the various events is involved in neuronal cell death. Treatment with G115 (the ginseng extract) reduce the accumulation of $\alpha$-synuclein aggregates and protect dopaminergic terminals in the striatum [29]. Irisflorentin, derived from the roots of Belamcanda chinensis (L.) DC, is an herb which has been used for the treatment of inflammatory disorders in TCM. Irisflorentin hinders $\alpha$-synuclein accumulation in the OW13 strain of C. elegans [30].

\section{Induce Autophagy}

$\alpha$-synuclein aggregates are toxic to neurons, however, the mechanism is still a controversial topic [31]. Aggregation and degradation of $\alpha$-synuclein is maintained in a dynamic balance under physiological conditions. Initial studies suggest that $\alpha$-synuclein is degraded via the UPS (ubiquitin-proteasome system). Further studies have shown that ALP (lysosomal autophagy pathway) is another major cell protein degradation pathway [32]. Autophagy modulation may be employed for therapeutic intervention during the maintenance of neurodegenerative disorders [33].

PIP (piperine) is a Chinese medicine with anti-inflammatory and antioxidant effects, exerts neuroprotective effects in PD models via induction of autophagy, and may be an effective agent for PD treatment [34]. PF (Paeoniflorin) is the principal bioactive component of Radix Paeoniae Alba, which is widely used in TCM for the treatment of neurodegenerative disorders, such as PD. Cao et al. [35] provided the first experimental evidence of $\mathrm{PF}$ modulation in models of neuron injury. Our findings [36] showed Paeoniflorin reduced CAT (catalase) and SOD (superoxide dismutase) activities, increased cell viability, and protected cells against oxidative stress caused by MPP+ (1-methyl-4-phenylpyridinium). In addition, Paeoniflorin significantly reduced the damage caused by MPP+, cells returned to normal state. Moreover, PF upregulated both autophagy and ubiquitin proteasome pathways. 


\section{Other Mechanisms and Multiple Effectiveness}

PD is a complicated disease, commonly diagnosed among the elderly, which leads to degeneration of the central nervous system. TCM has long been used to improve the treatment of PD by alleviating the toxic and adverse effects of Western drug-based intervention [37]. Experimental study of TCM treatment of PD more focused on the observation of drugs to improve neurobehavioral symptoms, the protection of dopaminergic nerve cells, and so on, involving many different angles and mechanisms. Saponins, an important group of bioactive plant natural products, are glycosides of triterpenoid or steroidal aglycones. Their diverse biological activities are ascribed to their different structures. Saponins have long been recognized as key ingredients in traditional Chinese medicine. Accumulated evidence suggests that saponins have significant neuroprotective effects including antioxidant, modulation of neurotransmitters, anti-apoptosis, anti-inflammation, attenuating $\mathrm{Ca}^{2+}$ influx, modulating neurotrophic factors, inhibiting tau phosphorylation, and regeneration of neural networks [38]. EAS (Extract of Acanthopanax senticosus harms) has neuroprotective effect on PD mice against dopaminergic neuronal damage. The therapeutic effect of EAS on PD may involve in regulating the tyrosine metabolism, mitochondrial beta-oxidation of long chain saturated fatty acids, fatty acid metabolism, methionine metabolism and sphingolipid metabolism. This study indicated that changed metabolites can be certainly recovered by EAS, and the treatment of EAS can be connected with the regulation of related metabolic pathways $[12,39]$.

\section{Conclusions and Future Perspectives}

In recent years, our research team has done a lot of related studies, in terms of neural protection mechanisms of TCM. PF protected cells against oxidative stress, upregulated both autophagy and ubiquitin proteasome pathways [35, 36]. Resveratrol upregulated the expression of SIRT1, restored lysosomal function, enhanced Ox-LDL-induced impaired autophagic flux, and promoted Ox-LDL degradation through the autophagy-lysosome degradation pathway [40]. Although recent studies on the neuroprotective effect of Chinese traditional medicine and monomers have made a lot of progress, it is still in its infancy and many problems need to be solved:

(1) separation and quality control of TCM material (chemical composition);

(2) some medicine monomer or active ingredient research in prevention and treatment of the disease, which is still at the stage of the cell model, while there are no animal model experiments and lack of clinical efficacy of further observation and verification;

(3) the role of TCM in the treatment of disease monomeric components of a single pathway, which can not play its synergies and overall adjustment advantage;

(4) a variety of effective components and monomer of TCM, which act through a variety of different mechanisms and in different time periods;

(5) systematic study on the mechanism and efficacy of TCM, Chinese medicine monomer, more monomer complexing agents through the application of modern molecular biology methods, that make its results be accepted by Western medicine and the international community.

There are already many Chinese pharmaceutical enterprises in the ongoing modernization of Chinese medicine production and research. For example, one of well-known enterprises in China has a lot of Chinese medicine monomer complexing agent production. They have developed a new drug called "ginkgo diterpene lactone meglumine injection", which has been approved for marketing at the end of the year 2012. Its main components are Ginkgolide A, Ginkgolide B, Ginkgolide K, etc., and it has a definite clinical efficacy [41]. No matter what therapeutic methods are, the effects need to be confirmed by strict 
and long-term animal experiments before clinical application.

\section{References}

[1] Bisaglia, M., Filograna, R., Beltramini, M., and Bubacco, L. 2014. "Are Dopamine Derivatives Implicated in the Pathogenesis of Parkinson's Disease." Ageing Res. Rev. 13: 107-14.

[2] Kalia, L. V., and Kalia, S. K. 2015. "Alpha-Synuclein and Lewy Pathology in Parkinson's Disease." Curr. Opin. Neurol. 28: 375-81.

[3] Tan, C. C., Yu, J. T., Tan, M. S., Jiang, T., Zhu, X. C., and Tan, L. 2014. "Autophagy in Aging and Neurodegenerative Diseases: Implications for Pathogenesis and Therapy." Neurobiol. Aging 35: 941-57.

[4] Bose, A., and Beal, M. F. 2016. "Mitochondrial Dysfunction in Parkinson's Disease." J. Neurochem. 139: 216-31.

[5] Bourdenx, M., Koulakiotis, N. S., Sanoudou, D., Bezard, E., Dehay, B., and Tsarbopoulos, A. 2015. "Protein Aggregation and Neurodegeneration in Prototypical Neurodegenerative Diseases: Examples of Amyloidopathies, Tauopathies and Synucleinopathies." Prog. Neurobiol. 2015: S0301-0082(15)00077-5.

[6] Rott, R., Szargel, R., Shani, V., Bisharat, S., and Engelender, S. 2014. "Alpha-Synuclein Ubiquitination and Novel Therapeutic Targets for Parkinson's Disease." CNS Neurol. Disord. Drug Targets 13: 630-37.

[7] Kalia, L. V., and Kalia, S. K. 2015. "Alpha-Synuclein and Lewy Pathology in Parkinson's Disease." Curr. Opin. Neurol. 28:375-81.

[8] Bisaglia, M., Filograna, R., Beltramini, M., and Bubacco, L. 2014. "Are Dopamine Derivatives Implicated in the Pathogenesis of Parkinson's Disease." Ageing Res. Rev. 13: 107-14.

[9] Wu, F., Li, H., Jin, L., Li, X., Ma, Y., You, J., et al. 2013. "Deer Antler Base as a Traditional Chinese Medicine: A Review of Its Traditional Uses, Chemistry and Pharmacology." J. Ethnopharmacol. 145: 403-15.

[10] Iwasaki, K., Kato, S., Monma, Y., Niu, K., Ohrui, T., Okitsu, R., et al. 2007. "A Pilot Study of Banxia Houpu Tang, a Traditional Chinese Medicine, for Reducing Pneumonia Risk in Older Adults with Dementia." J. Am. Geriatr. Soc. 55: 2035-40.

[11] Zhang, G., Xiong, N., Zhang, Z., Liu, L., Huang, J., Yang, J., et al. 2015. "Effectiveness of Traditional Chinese Medicine as an Adjunct Therapy for Parkinson's Disease: A Systematic Review and Meta-analysis." PLoS One 10: e0118498.

[12] Li, X. Z., Zhang, S. N., Lu, F., Liu, C. F., Wang, Y., Bai, Y., et al. 2013. "Cerebral Metabonomics Study on
Parkinson's Disease Mice Treated with Extract of Acanthopanax senticosus Harms." Phytomedicine 20: 1219-29.

[13] Jia, L., Zhao, Y., and Liang, X. J. 2009. Current Evaluation of the Millennium Phytomedicine-Ginseng (II): Collected Chemical Entities, Modern Pharmacology, and Clinical Applications Emanated from Traditional Chinese Medicine." Curr. Med. Chem. 16: 2924-42.

[14] Gao, J. W., Yamane, T., Maita, H., Ishikawa, S., Iguchi-Ariga, S. M., Pu, X. P., et al. 2011. "DJ-1-Mediated Protective Effect of Protocatechuic Aldehyde against Oxidative Stress in SH-SY5Y Cells." J. Pharmacol. Sci. 115:36-44.

[15] Koppula, S., Kumar, H., More, S. V., Kim, B. W., Kim, I. S., and Choi, D. K. 2012. "Recent Advances on the Neuroprotective Potential of Antioxidants in Experimental Models of Parkinson's Disease." Int. J. Mol. Sci. 13:10608-29.

[16] Kumar, H., Lim, H. W., More, S. V., Kim, B. W., Koppula, S., Kim, I. S., et al. 2012. "The Role of Free Radicals in the aging Brain and Parkinson's Disease: Convergence and Parallelism." Int. J. Mol. Sci. 13:10478-504.

[17] Chan, W. S., Durairajan, S. S., Lu, J. H., Wang, Y., Xie, L. X., Kum, W. F., et al. 2009. "Neuroprotective Effects of Astragaloside IV in 6-hydroxydopamine-treated Primary Nigral Cell Culture.” Neurochem. Int. 55: 414-22.

[18] Gao, J. W., Yamane, T., Maita, H., Ishikawa, S., Iguchi-Ariga, S. M., Pu, X. P., et al. 2011. "DJ-1-Mediated Protective Effect of Protocatechuic Aldehyde against Oxidative Stress in SH-SY5Y Cells." $J$. Pharmacol. Sci. 115: 36-44.

[19] Tseng, Y. T., Chang, F. R., and Lo, Y. C. 2014. "The Chinese Herbal Formula Liuwei Dihuang Protects Dopaminergic Neurons against Parkinson's Toxin through Enhancing Antioxidative Defense and Preventing Apoptotic Death." Phytomedicine 21: 724-33.

[20] Radi, E., Formichi, P., Battisti, C., and Federico, A. 2014. "Apoptosis and Oxidative Stress in Neurodegenerative Diseases.” J. Alzheimers Dis. 42: S125-52.

[21] Chen, Y., Zhang, Y., Li, L., and Holscher, C. 2015. "Neuroprotective Effects of Geniposide in the MPTP Mouse Model of Parkinson's Disease." Eur. J. Pharmacol. 768: 21-7.

[22] Zhang, Y., Xia, Z., Liu, J., and Yin, F. 2015. "Cell Signaling Mechanisms by which Geniposide Regulates Insulin-Degrading Enzyme Expression in Primary Cortical Neurons." CNS Neurol. Disord. Drug Targets 14: 370-77.

[23] Chen, J. Ma, J., Qiu, Y., Yi, S., Liu, Y., Zhou, Q., et al. 2012. "Effects of Zhichan Powder on Signal Transduction and Apoptosis-Associated Gene Expression in the 
Substantia Nigra of Parkinson's Disease Rats." Neural. Regen. Res. 7: 2115-22.

[24] Procaccio, V., Bris, C., Chao de la Barca, J. M., Oca, F., Chevrollier, A., Amati-Bonneau, P., et al. 2014. "Perspectives of Drug-Based Neuroprotection Targeting Mitochondria." Rev. Neurol. (Paris) 170: 390-400.

[25] Sun, R., Wang, K., Wu, D., Li, X., and Ou, Y. 2012. "Protective Effect of Paeoniflorin against Glutamate-Induced Neurotoxicity in PC12 Cells via Bcl-2/Bax Signal Pathway." Folia Neuropathol. 50: 270-76.

[26] Zhang, Y., Gonga, X. G., Wang, Z. Z., Suna, H. M., Guoa, Z. Y., Gaia, C. et al. 2016. "Protective Effects of DJ-1 Medicated Akt Phosphorylation on Mitochondrial Function Are Promoted by Da-Bu-Yin-Wan in 1-methyl-4-phenylpyridinium-treated Human Neuroblastoma SH-SY5Y Cells.” J. Ethnopharmacol. 187: 83-93.

[27] Wang W, Nguyen, L.T., Burlak, C., Chegini, F., Guo, F., Chataway, T., et al. 2016. "Caspase-1 Causes Truncation and Aggregation of the Parkinson's Disease-Associated Protein Alpha-Synuclein." Proc. Natl. Acad. Sci. USA 113: 9587-92.

[28] Stefanis, L. 2012. "Alpha-Synuclein in Parkinson's Disease.” Cold Spring Harb. Perspect. Med. 2: a009399.

[29] Van Kampen, J. M., Baranowski, D. B., Shaw, C. A., and Kay, D. G. 2014. "Panax Ginseng is Neuroprotective in a Novel Progressive Model of Parkinson's Disease.” Exp. Gerontol. 50: 95-105.

[30] Chen, Y. M., Liu, S. P., Lin, H. L., Chan, M. C., Chen, Y. C., Huang, Y. C., et al. 2015. "Irisflorentin Improves Alpha-Synuclein Accumulation and Attenuates 6-OHDA-induced Dopaminergic Neuron Degeneration, Implication for Parkinson's Disease Therapy." Biomedicine (Taipei) 5: 4.

[31] Xu, W., Tan, L., and Yu, J. T. 2015. "Link between the SNCA Gene and Parkinsonism." Neurobiol. Aging 36: 1505-18

[32] Martinez-Vicente, M. 2012. "Multiple Ways for a-Synuclein Degradation." Mov. Disord. 27: 345.

[33] Ghavami, S., Shojaei, S., Yeganeh, B., Ande, S. R.,
Jangamreddy, J. R., Mehrpour, M., et al. 2014. "Autophagy and Apoptosis Dysfunction in Neurodegenerative Disorders." Prog. Neurobiol. 112: 24-49.

[34] Liu, J., Chen, M., Wang, X., Wang, Y., Duan, C., Gao, G., et al. 2016. "Piperine Induces Autophagy by Enhancing Protein Phosphotase 2A Activity in a Rotenone-Induced Parkinson's Disease Model." Oncotarget. 2016 August: 27. doi: 10.18632/oncotarget.11661.

[35] Cao, B. Y., Yang, Y. P., Luo, W. F., Mao, C. J., Han, R., Sun, X., et al. 2010. "Paeoniflorin, a Potent Natural Compound, Protects PC12 Cells from MPP+ and Acidic Damage via Autophagic Pathway." J. Ethnopharmacol. 131: 122-9.

[36] Cai Z, Zhang, X., Zhang, Y., Li, X., Xu, J., and Li, X. 2015. "The Impact of Paeoniflorin on Alpha-Synuclein Degradation Pathway." Evid. Based Complement Alternat. Med. 2015: 1-8.

[37] Zhang, J., Ma, Y. Z., and Shen, X. M. 2013. "Evaluation on the Efficacy and Safety of Chinese Herbal Medication Xifeng Dingchan Pill in Treating Parkinson's Disease: Study Protocol of a Multicenter, Open-Label, Randomized Active-Controlled Trial.” J. Integr. Med. 11: 285-90.

[38] Sun, A., Xu, X., Lin, J., Cui, X., and Xu, R. 2015. "Neuroprotection by Saponins." Phytother. Res. 29: 187-200.

[39] Li, X. Z., Zhang, S. N., Wang, K. X., Liu, H. Y., Yang, Z. M., Liu, S. M., et al. 2014. "Neuroprotective Effects of Extract of Acanthopanax Senticosus Harms on SH-SY5Y Cells Overexpressing Wild-Type or A53T Mutant Alpha-Synuclein." Phytomedicine 21: 704-11.

[40] Zhang, Y., Cao, X., Zhu, W., Liu, Z., Liu, H., Zhou, Y., et al. 2016. "Resveratrol Enhances Autophagic Flux and Promotes Ox-LDL Degradation in HUVECs via Upregulation of SIRT1." Oxidative Medicine and Cellular Longevity 2016: 7589813.

[41] Wang, S., Ouyang, B., Aa, J., Geng, J., Fei, F., Wang, P., et al. 2016. "Pharmacokinetics and Tissue Distribution of Ginkgolide A, Ginkgolide B, and Ginkgolide K after Intravenous Infusion of Ginkgo Diterpene Lactones in a Rat Model." J. Pharm. Biomed. Anal. 126: 109-16. 